\title{
Stark Ladders of Resonances: Wannier Ladders and Perturbation Theory
}

\author{
Vincenzo Grecchi ${ }^{1}$, Marco Maioli ${ }^{2}$, Andrea Sacchetti ${ }^{2,3}$ \\ I Dipartimento di Matematica, Università di Bologna, I-40127 Bologna, Italy \\ e-mail: Grecchi@dm unibo it \\ 2 Dipartimento di Matematica, Università di Modena, I-41100 Modena, Italy \\ 3 e-mail: Sacchetti@imovx2 unimo.it
}

Received: 27 January 1993/in revised form: 30 April 1993

\begin{abstract}
Let $H_{B}$ be any fixed one-dimensional Bloch Hamiltonian with only the first $m$ gaps open and $H_{F}=H_{B}+F x$ be the corresponding Stark Hamiltonian. For any positive $F$ small enough $H_{F}$ has only $m$ ladders of sharp resonances given by the analytic translation method, the decoupled band approximation and the regular perturbation theory. This way, the Wannier conjecture becomes a definite regular perturbation theory for the Stark ladders as eigenvalues of the translated Hamiltonian.
\end{abstract}

\section{Introduction}

In 1960 Wannier [23] suggested the existence of (Stark) ladders of bound states (or resonances) in the Bloch Stark Hamiltonian:

$$
H_{F}=-\hbar^{2} \frac{d^{2}}{d x^{2}}+V(x)+F x, \quad V(x)=V(x+a) \quad \text { and } \quad F, a>0,
$$

which does not have any bound state for zero external electric field. This location effect given by the external uniform field on Bloch problems is in agreement with the tilted bands picture of Zener. Actually Wannier proved that the one band approximation gives a (Wannier) ladder of bound states for each finite band, but he was not able to extend the result to the full problem. Even in the absence of definite experimental results, the existence of the ladders was put in doubt [24]. Some years ago this attitude changed because of the accurate numerical works $[4,6]$, the new experimental results [3] and a rigorous definition of ladder resonances by means of eigenvalues of a suitable operator [15]. In 1982 Avron [2], assuming the existence of ladders of resonances and using the crystal momentum representation, studied the width behavior of the resonances in the Fermi Golden Rule approximation. Recently, rigorous proofs of existence have appeared in different regimes: for large electric field strength [1], for electric field strength small with $\hbar[8,10]$ and for large period [13].

We want now to continue and to extend the line of research started with Wannier in order to obtain the existence of resonances for a fixed Bloch model and weak 
external field. In this paper, making the assumption of a finite number $m$ of finite bands as in Avron [2], we prove the existence of ladders of resonances for $a$ and $\hbar$ fixed and arbitrarily small electric field strength $F$. Since the potential is translation analytic, as in Herbst and Howland [15] we consider the translated Hamiltonian

$$
H_{F}^{\lambda}=-\frac{d^{2}}{d x^{2}}+V(x+\lambda)+F x+F \lambda ; \quad \Im \lambda<0,
$$

and we extend the crystal momentum representation and the Wannier decoupled band (DB) approximation to the case of complex $\lambda$. The spectrum of the Wannier DB approximation is the union of $m$ ladders of real eigenvalues and the line $\lambda F+\mathrm{R}$. Moreover, the coupling term between the bands is relatively compact with respect to the Wannier DB approximation. Therefore, the Wannier ladders become ladders of complex eigenvalues of (I.2) (resonances of (I.1)) when the coupling term between the bands is restored. Our proof of existence, based on the new extension of the crystal momentum representation to the analytic translated problem, justifies the Wannier DB aproximation as the basis of a regular perturbation theory.

We have now the first part of a rigorous theory on the Stark ladders containing all the already known results as the asymptotic expansion of Nenciu [7, 12, 19, 21], the Buslaev-Dmitrieva [9] asymptotic behavior of the widht of the last ladder and the generic singular behavior of the ladders given by us [12]. We give here the explicit exponential behavior of the width of the last ladder up to the second perturbation order (Fermi Golden Rule) previously studied by Avron [2]. Another interesting analysis should regard the behavior of the crossings of different ladders considered by Avron [2], Bentosela et al. [6], and Ferrari et al. [11].

Let us notice that the class of potentials with a finite number of bands is not void and moreover it is dense in the set of real-valued $L_{\text {loc }}^{2}$ periodic potentials [18]. So, the general problem could be seen as a limit case of the one considered here. The results of this paper have been announced in [14]. In Sect. 2 of this paper we define the extended crystal momentum representation (ECMR) for the translated Bloch operator in the case of a finite number of bands. In Sect. 3 we define the resonances and we prove the existence of them by means of the regular perturbation theory and the Kato-Rellich Theorem. Finally, in Sect. 4 we give the behavior of the width for the second order expansion.

\section{The Extended Crystal Momentum Representation (ECRM) for the Finite Bands Number Model}

Let us consider the one-dimensional Bloch Hamiltonian $H_{B}$ with real-valued $L_{\text {loc }}^{2}(\mathrm{R})$ periodic potential $V(x)$ of period $2 \pi$ :

$$
H_{B}=-\frac{d^{2}}{d x^{2}}+V(x)
$$

The lower bounded self-adjoint operator formally defined by $H_{B}$ has purely absolutely continuous spectrum which is the union of closed intervals, called bands, separated by open intervals, called gaps [20]. In the following we shall assume:

Hypothesis. The spectrum of the Bloch operator $H_{B}$ has a finite number of bands, say $m+1$ More precisely, we assume that the first $m$ gaps are open and the other ones closed, that is.

$$
\sigma\left(H_{B}\right)=\left[E_{1}^{b}, E_{1}^{t}\right] \cup \ldots \cup\left[E_{m}^{b}, E_{m}^{t}\right] \cup\left[E_{m+1}^{b},+\infty\right), \quad E_{l}^{t}<E_{l+1}^{b}, l=1, \ldots, m
$$


Hence, see Theorem XIII.91d in [20], the periodic potential $V(x)$ is analytic in the stripe $|\Im x|<2 \lambda_{0}+\delta$, for some $\lambda_{0}>0$ and $\delta>0$.

In this section we define the extended crystal momentum representation (ECRM) for the operator formally defined by

$$
H_{F}^{\lambda}=H_{B}^{\lambda}+F x+F \lambda, \quad H_{B}^{\lambda}=-\frac{d^{2}}{d x^{2}}+V(x+\lambda), \quad|\Im \lambda|<2 \lambda_{0}+\delta
$$

In order to do this we need the following results on the band functions of $H_{B}$. The energy function $\mathscr{E}(p)$ is a multisheeted analytic function in the complex plane with branch points of square root type $\pm z_{l}$ and $\pm \bar{z}_{l}$, where $z_{l}=l / 2+i h_{l}, l=1,2, \ldots, m$ and $h_{l}>0$. The restrictions of $\mathscr{E}(p)$ on particular sheets give the band functions $\mathscr{E}_{l}(k), l=1, \ldots, m$, and $\mathscr{E}_{m+1}(p)$ (Theorem XIII.95 in [20]). The firsts $m$ band functions $\mathscr{E}_{l}(k)$ are periodic functions of period 1 and

$$
E_{l}^{b}=\min _{k \in \mathscr{B}} \mathscr{E}_{l}(k), \quad E_{l}^{t}=\max _{k \in ß} \mathscr{E}_{l}(k)
$$

where $\mathscr{S}$ is the Brillouin zone, that is the torus $\mathrm{R} / 1$ with respresentatives on $(0,1]$. The $m+1$-th band function $\mathscr{E}_{m+1}(p)$ is defined by $\mathscr{E}(p)$ on the sheet obtained by cutting the complex plane by two lines linking directly $\frac{m}{2}+i \infty$ and $\frac{m}{2}-i \infty$ with, respectively, $z_{m}$ and $\bar{z}_{m}$ and such that

$$
\mathscr{E}(m / 2)=\mathscr{E}_{m+1}(m / 2)=E_{m+1}^{b} .
$$

Let $\varphi_{l}(x, k)=e^{i k x} u_{l}(x, k)$ and $\varphi_{m+1}(x, p)=e^{i p x} u_{m+1}(x, p)$ be the Bloch functions associated to the band functions, where $u_{l}(x, k)$ and $u_{m+1}(x, p)$ are periodic functions in $x$ of period $2 \pi$ and, for fixed $x, \varphi_{l}(x, k)$ are periodic functions on the Brillouin zone $\mathscr{B}$. We have this result (in the following let us denote by $C$ any positive constant, $\partial_{k}:=\frac{\partial}{\partial k}$ and $\left.\partial_{p}:=\frac{\partial}{\partial p}\right)$.

Lemma 1. Let $\mathbf{w}_{l}^{k}=\left\{w_{l}^{k}(K)\right\}_{K \in Z}$ and $\mathbf{w}_{m+1}^{p}=\left\{w_{m+1}^{p}(K)\right\}_{K \in Z}$, be, respectively, the Fourier coefficients of $u_{l}(x, k)$ and $u_{m+1}(x, p)$. Then, the following estimates uniformly hold for $p \in \mathrm{R}$ and $K \in \mathrm{Z}$.

$$
\left|w_{l}^{p}(K)\right| \leq C \exp \left[-2|K+p| \lambda_{0}\right], \quad\left|\partial_{p} w_{l}^{p}(K)\right| \leq C \exp \left[-2|K+p| \lambda_{0}\right],
$$

for any $l, l=1, \ldots, m$, and

$$
\begin{gathered}
\left|w_{m+1}^{p}(K)\right| \leq C \exp \left[-2|K| \lambda_{0}\right], \\
\left|\partial_{p} w_{m+1}^{p}(K)\right| \leq C(1+|p|)^{-2} \exp \left[-2|K| \lambda_{0}\right],
\end{gathered}
$$

for some positive constant $C$.

Proof From the hypothesis we have that for any fixed $k \in \mathscr{B}$ the periodic functions $u_{l}(x, k)$ and their derivatives $\partial_{k} u_{l}(x, k)$ are analytic functions for $x$ in the stripe $|\Im x|<2 \lambda_{0}+\delta$. Therefore they are uniformly bounded for $k \in \mathscr{B}$ and $|\Im x| \leq 2 \lambda_{0}$ and so, from the well known estimate on the Fourier coefficients of analytic functions, we have for $k \in \mathscr{P}$ and $K \in \mathrm{Z}$ :

$$
\left|w_{l}^{k}(K)\right| \leq C \exp \left[-2|K| \lambda_{0}\right] \quad \text { and } \quad\left|\partial_{p} w_{l}^{k}(K)\right| \leq C \exp \left[-2|K| \lambda_{0}\right] .
$$


Moreover, taking into account that $w_{l}^{(k+1)}(K)=w_{l}^{k}(K+1)$, (II.3) follows. Now, in order to prove (II.4), the same arguments still work since the uniform boundedness of $u_{m+1}(x, p)$ and of its derivative $\partial_{p} u_{m+1}(x, p)$ for $x$ in the stripe $|\Im x| \leq 2 \lambda_{0}$ and $p \in \mathrm{R}$ follows from the asymptotic behaviors

$$
u_{m+1}(x, p)=1+O\left(p^{-1}\right) \quad \text { and } \quad \partial_{p} u_{m+1}(x, p)=O\left(p^{-2}\right)
$$

as $p$ goes to infinity. See, for instance, Sect. 21.7 in [22] for $x \in[0,2 \pi]$ and in the case of $x+i \alpha, \alpha \in\left[-2 \lambda_{0},+2 \lambda_{0}\right]$, (II.6) follows in a similar way. In particular, we remark that the estimates 21.7 .2 and 21.7.3 in [22] hold for any $p$ because in the infinite band the singular crossings are at a finite distance $h_{m}$ from the real axis.

Finally, we have the completeness result for the Bloch functions (Theorem XIII.98 in [20]), that, for such a finite number bands model, assumes the following form: let $f \in \mathscr{Y}(\mathrm{R}), \mathscr{S}$ is the space of functions of rapid decrease, and let

$$
\xi_{l}(k)=\frac{1}{\sqrt{2 \pi}} \int_{\mathrm{R}} f(x) \overline{\varphi_{l}(x, k)} d x \quad \text { and } \quad \eta(p)=\frac{1}{\sqrt{2 \pi}} \int_{\mathrm{R}} f(x) \overline{\varphi_{m+1}(x, p)} d x .
$$

Then, we have that

$$
\begin{aligned}
f(x)= & \sum_{l=1}^{m} \frac{1}{\sqrt{2 \pi}} \int_{\mathcal{B}} \xi_{l}(k) \varphi_{l}(x, k) d k \\
& +\frac{1}{\sqrt{2 \pi}} \int_{\mathrm{R}} \eta(p) \varphi_{m+1}(x, p) d p
\end{aligned}
$$

and

$$
\|f\|_{L^{2}(\mathrm{R}, d x)}^{2}=\sum_{l=1}^{m}\left\|\xi_{l}\right\|_{L^{2}(\not \beta, d k)}^{2}+\|\eta\|_{L^{2}(\mathrm{R}, d p)}^{2} .
$$

By extending this isometric transformation on $L^{2}(\mathrm{R}, d x)$ by continuity we have a unitary transformation $(\xi, \eta)=\mathbb{Q} f, \xi=\left(\xi_{1}, \ldots, \xi_{m}\right)$, which maps $L^{2}(\mathrm{R}, d x)$ onto $\mathscr{H}_{h}=\mathscr{H}_{d} \oplus \mathscr{H}_{c}$, where $\mathscr{H}_{d}=\bigoplus_{l=1}^{m} \mathscr{H}_{l}, \mathscr{H}_{l}=L^{2}(\mathscr{B}, d k)$ and $\mathscr{H}_{c}=L^{2}(\mathrm{R}, d p)$.

We consider now the translated Bloch operator $H_{B}^{\lambda}$. The band functions of $H_{B}^{\lambda}$ coincides with the old ones and the associated Bloch functions are given by $\varphi_{l}(x+\lambda, k)$ and $\varphi_{m+1}(x+\lambda, p)$. The above completeness result (II.7) and (II.8) takes the following form for the Bloch functions of $H_{B}^{\lambda}$ ):

Theorem 2. Let $\mathscr{D}$ the dense space spanned by the parabolic cylinder functions $D_{n}(x)=e^{-x^{2} / 2} H_{n}(x), n=0,1, \ldots$ The transformation $\mathscr{Z}^{\lambda}: L^{2}(\mathrm{R}, d x) \rightarrow \mathscr{H}$ defined on $\mathscr{D}$ by $(\xi, \eta)=\mathscr{Q}^{\lambda} f$, where

$$
\begin{aligned}
\xi_{l}(k) & =\frac{1}{\sqrt{2 \pi}} \int_{\mathrm{R}} f(x) \overline{\varphi_{l}(x+\bar{\lambda}, k)} d x, \\
\eta(p) & =\frac{1}{\sqrt{2 \pi}} \int_{\mathrm{R}} f(x) e^{-i p x} \overline{u_{m+1}(x+\bar{\lambda}, p)} d x,
\end{aligned}
$$


and such that:

$$
\begin{aligned}
f(x)= & \sum_{l=1}^{m} \frac{1}{\sqrt{2 \pi}} \int_{\not \supset} \xi_{l}(k) \varphi_{l}(x+\lambda, k) d k \\
& +\frac{1}{\sqrt{2 \pi}} \int_{\mathrm{R}} \eta(p) e^{\imath p x} u_{m+1}(x+\lambda, p) d p,
\end{aligned}
$$

admit a bounded analytic extension $\mathbb{l}^{\lambda}$ for $\lambda$ in the stripe $|\Im \lambda|<2 \lambda_{0}$, unitary for $\lambda$ real and with bounded analytic inverse $\left(\mathbb{Z}^{\lambda}\right)^{-1}$

Proof For real $\lambda, t^{\lambda} f$ coincides, up to a phase factor, with the previous transformation $\#$ of $f(x-\lambda)$ wich unitarily maps $L^{2}(\mathrm{R}, d x)$ onto . $\mathscr{H}$. Let $\lambda \in \mathrm{C}$ and

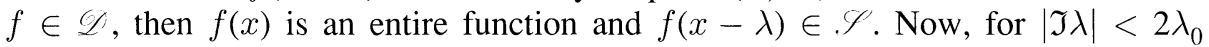
(II.10) and (II.11) follow computing (II.7) and (II.8) for $f(x-\lambda)$ and redefining $\eta(p)$ by $\eta(p) \rightarrow e^{i p \lambda} \eta(p)$. Boundedness and analyticity of $2 b^{\lambda}$ follow from (II.10) and Lemma 1. Indeed, one has

$$
\eta(p)=\sum_{K \in \mathrm{Z}} e^{-\imath K \lambda} \overline{w_{m+1}^{p}(K)} \hat{f}(p+K),
$$

where the Fourier coefficients $w_{m+1}^{p}(K)$ of $u_{m+1}(x, p)$ satisfy the estimates (II.4), hence

$$
\|\eta\|_{\dddot{\#}_{c}} \leq C\|\hat{f}\| \sum_{K \in \mathrm{Z}} \exp \left[K \Im \mathfrak{I} \lambda-2|K| \lambda_{0}\right] \leq C\|f\|_{L^{2}(\mathrm{R}, d x)} .
$$

Since the same results still hold for any $\xi_{l}(k)$, boundedness and analyticity of $2 l^{\lambda}$ follow. In a similar way, from (II.11) and Lemma 1, boundedness and analyticity of the inverse $\left(\mathbb{l}^{\lambda}\right)^{-1}$ follow too.

Here, we call the vector $(\xi, \eta)=\mathscr{\not l}^{\lambda} f$ of $\mathscr{H}_{,} \xi=\left(\xi_{1}, \ldots, \xi_{m}\right) \in \mathscr{H}_{d}$ and $\eta \in \mathscr{H}_{c}$, the extended crystal momentum representation (ECMR) corresponding to the vector $f \in L^{2}(\mathrm{R}, d x)$. In this representation the position operator $x$ takes the form

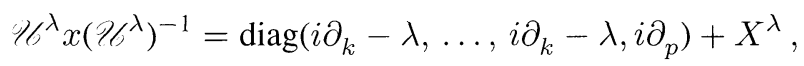

where $X^{\lambda}(\xi, \eta)=\mathbb{q}^{\lambda} w^{\lambda}$ and

$$
\begin{aligned}
w^{\lambda}(x)= & \frac{i}{\sqrt{2 \pi}}\left[\sum_{l=1}^{m} \int_{\Re} \xi_{l}(k) e^{\imath k(x+\lambda)} \partial_{k} u_{l}(x+\lambda, k) d k\right. \\
& \left.+\int_{\mathrm{R}} \eta(p) e^{\imath p x} \partial_{p} u_{m+1}(x+\lambda, p) d p\right] .
\end{aligned}
$$

Theorem 3. $X^{\lambda}$ is an analytic family of bounded operators in the stripe $|\Im \lambda|<2 \lambda_{0}$. Proof From the boundedness of $7^{\lambda}$ we have

$$
\left\|X^{\lambda}(\xi, \eta)\right\|_{\not H} \leq\left\|\mathbb{Z}^{\lambda}\right\| \cdot\left\|w^{\lambda}\right\|_{L^{2}(\mathrm{R}, d x)}
$$


and, from (II.13) and Lemma 1, boundedness of $X^{\lambda}$ follows; indeed:

$$
\begin{aligned}
\left\|w^{\lambda}\right\|_{L^{2}(\mathrm{R}, d x)} \leq & \sum_{l=1}^{m}\left\|\xi_{l}\right\|_{\mathscr{H}_{l}} \sum_{K \in \mathrm{Z}}\left\|e^{i(k+K) \lambda} \partial_{k} w_{l}^{k}(K)\right\|_{\mathscr{H}_{l}} \\
& +\|\eta\|_{\mathscr{H}_{c}} \sum_{K \in \mathrm{Z}}\left\|e^{i K \lambda} \partial_{p} w_{m+1}^{p}(K)\right\|_{\mathscr{H}_{c}} \leq C\|(\xi, \eta)\|_{\mathscr{H}} .
\end{aligned}
$$

Analyticity of the family $X^{\lambda}$ follows immediately from Theorem 2 and (II.13).

Remark 4. Let us stress that this result does not extend to the general case of an analytic potential with infinitely many open gaps.

Here, the operator $X^{\lambda}$ on $\mathscr{H}_{b}=\mathscr{H}_{d} \oplus \mathscr{H}_{c}$ represents the coupling term between all the bands and it has the form

$$
X^{\lambda}=\left(\begin{array}{ll}
X_{d, d}^{\lambda} & X_{d, c}^{\lambda} \\
X_{c, d}^{\lambda} & X_{c, c}^{\lambda}
\end{array}\right) .
$$

More precisely, the term $X_{d, d}^{\lambda}$ coincides with the usual interband matrix which couples the first $m$ bands, that is:

$$
X_{d, d}^{\lambda}=\left(X_{d, d}^{l, l^{\prime}}\right)_{l, l^{\prime}},
$$

where

$$
X_{d, d}^{l, l^{\prime}}(k)=i\left(\partial_{k} \mathbf{w}_{l}^{k}, \mathbf{w}_{l^{\prime}}^{k}\right)_{l^{2}(\mathrm{Z})}:=i \sum_{K \in Z} \partial_{k} w_{l}^{k}(K) \overline{w_{l^{\prime}}^{k}(K)} .
$$

The term $X_{c, c}^{\lambda}$ couples the infinite band with itself and it is given by

$$
\begin{aligned}
\left(X_{c, c}^{\lambda} \eta\right)(p) & =\sum_{L \in Z} e^{-i L \lambda} X_{c, c}^{L}(p) \eta(p+L), \\
X_{c, c}^{L}(p) & =i\left(\partial_{p} \mathbf{w}_{m+1}^{(p+L)}, T_{L} \mathbf{w}_{m+1}^{p}\right)_{l^{2}(\mathrm{Z})},
\end{aligned}
$$

where $T_{L}$ is the translation operator in $l^{2}(\mathrm{Z}):\left(T_{L} \mathbf{a}\right)(K)=a(K+L)$. Now, since $\varphi_{l}(x, k)$ and $\varphi_{m+1}(x, p)$ are orthonormal functions in $L^{2}([0,2 \pi], d x / 2 \pi)$, we have that:

$$
X_{d, d}^{l, l^{\prime}}(k)=\overline{X_{d, d}^{l^{\prime}, l}(k)} \quad \text { and } \quad X_{l}(k):=X_{d, d}^{l, l}(k) \equiv \overline{X_{l}(k)} \quad \text { for any } k
$$

and

$$
X_{c, c}^{-L}(p)=\overline{X_{c, c}^{L}(p-L)} \text { for any } p .
$$

Moreover, by the gauge choice $u_{m+1}(x, p) \rightarrow e^{i \phi_{m+1}(p)} u_{m+1}(x, p)$, we can choose $X_{c, c}^{0}(p)$ identically zero for a real and suitable $\phi_{m+1}(p)$. Therefore, in (II.16) the sum is taken on $\mathrm{Z}-\{0\}$. let us stress that, in general, one can choose $X_{l}(k)$ constant by the gauge choice $u_{l}(x, k) \rightarrow e^{\imath \phi_{l}(k)} u_{l}(x, k)$ for a real suitable function $\phi_{l}$ defined on $\mathscr{S}$; in particular, if the potential is even or odd, $X_{l}$ can be chosen identically zero for each $l$. Finally, $X_{d, c}^{\lambda}$ and $X_{c, d}^{\lambda}$ are given by:

$$
\begin{aligned}
\left(X_{c, d}^{\lambda} \xi\right)(p) & =\sum_{l=1}^{m} e^{\imath p \lambda} X_{c, d}^{l}(p) \xi_{l}(p), \quad X_{c, d}^{l}(p)=i\left(\partial_{p} \mathbf{w}_{l}^{p}, \mathbf{w}_{m+1}^{p}\right)_{l^{2}(\mathrm{Z})} \\
\left(X_{d, c}^{\lambda} \eta\right)_{l}(k) & =\sum_{L \in \mathrm{Z}} e^{-i(k+L) \lambda} X_{d, c}^{l}(k+L) \eta(k+L), \quad X_{d, c}^{l}(p)=\overline{X_{c, d}^{l}(p)} .
\end{aligned}
$$


Remark 5. From Lemma 1 the exponentially decreasing behavior follows:

$$
\begin{aligned}
& \left|X_{c, d}^{l}(p)\right| \leq C|p| \exp \left[-2|p| \lambda_{0}\right] \text { for any } l \\
& \left|X_{c, c}^{L}(p)\right| \leq C \cdot|L| \cdot(1+|p|)^{-2} \exp \left[-2|L| \lambda_{0}\right] .
\end{aligned}
$$

In the ECMR the operator corresponding to (I.2) is given by

$$
H_{F}^{\lambda}=H_{F}^{\mathrm{DB}, \lambda}+F \tilde{X}^{\lambda},
$$

where

$$
\begin{gathered}
H_{F}^{\mathrm{DB}, \lambda}=\operatorname{diag}\left(H_{1}, \ldots, H_{m}, H_{c}^{\lambda}\right) \\
\tilde{X}^{\lambda}:=X^{\lambda}-\operatorname{diag}\left(X_{\text {diag }}, 0\right) \quad \text { and } \quad X_{\text {diag }}=\operatorname{diag}\left(X_{1}, \ldots, X_{m}\right) .
\end{gathered}
$$

We dub $H_{F}^{\mathrm{DB}, \lambda}$ the decoupled band DB approximation and it acts on $\mathscr{H}$ as:

$$
H_{l}=i F \partial_{k}+\mathscr{E}_{l}+F X_{l}, \quad l=1,2, \ldots, m, \text { and } H_{c}^{\lambda}=i F \partial_{p}+\mathscr{E}_{m+1}+F \lambda \text {. }
$$

\section{Existence of the Stark Ladders and Perturbation Theory}

In order to prove the existence of resonances for the Stark-Wannier operator (I.1) we consider the family of operators (II.19) which is an analytic family of type (A) for $|\Im \lambda|<2 \lambda_{0}$. As we shall see, for $-\lambda_{0}<\mathfrak{I} \lambda<0$ the analytic translation $x \rightarrow x+\lambda$ defines the resonances by shifting the continuous spectrum of $H_{F}$ on the line $F \lambda+\mathrm{R}$ in the lower complex half-plane: they are the $\lambda$-independent eigenvalues of $H_{F}^{\lambda}$ between its essential spectrum and the real axis. Existence of them will follow by means of a stability result of the Wannier ladders with respect to the interband term.

Now, let us consider

$$
H_{F}^{\lambda}(f)=H_{F}^{\mathrm{DB}, \lambda}+f \tilde{X}^{\lambda},
$$

where $f \in \mathrm{C}$ is an auxiliary parameter which plays the role of the perturbative one in the perturbation theory; for $f=F$ we have, of course, $H_{F}^{\lambda}(F)=H_{F}^{\lambda}$. Here, $H_{F}^{\lambda}(f)$ is an analytic family of type (A) with respect to the parameters $f$ and $\lambda$. The resolvent $\left[H_{F}^{\lambda}(f)-z\right]^{-1}=R_{F}^{\lambda}(f, z) \equiv R^{\lambda}(f, z)$ (for sake of simplicity let us drop the parameter $F$ ) acts on $\mathscr{H}_{b}=\mathscr{H}_{d} \oplus \mathscr{H}_{c}$ as:

$$
R^{\lambda}(f, z)=\left(\begin{array}{ll}
R_{d}^{\lambda}(f, z) & S^{\lambda}(f, z) \\
T^{\lambda}(f, z) & R_{c}^{\lambda}(f, z)
\end{array}\right),
$$

where $R_{d}^{\lambda}(f, z)$ and $R_{c}^{\lambda}(f, z)$ are the compression of the resolvent on the subspace $\mathscr{H}_{d}$ and $\mathscr{H}_{c}$ : that is $R_{d}^{\lambda}(f, z)=\mathscr{P} R^{\lambda}(f, z) \mathscr{P}$ and $R_{c}^{\lambda}(f, z)=\mathscr{P}^{\perp} R^{\lambda}(f, z) \mathscr{P}^{\perp}$, where $\mathscr{P}$ is the projection on $\mathscr{H}_{d}$ and $\mathscr{P}^{\perp}=1-\mathscr{P}$ is the projection on $\mathscr{H}_{c}$. Moreover $R_{c}^{\lambda}(0, z)=\left[H_{c}^{\lambda}-z\right]^{-1}$ and $R_{d}(0, z)=\left[H_{d}-z\right]^{-1}$ are, respectively, the projection of the resolvent of the DB approximation on $\mathscr{H}_{c}$ and $\mathscr{H}_{d}$.

We have the following results:

Theorem 6. The spectrum of $H_{d}$ is purely discrete, $\lambda$-independent and coincides with the Wannier ladders:

$$
\sigma\left(H_{d}\right)=\left\{E_{l, j}=\left\langle\mathscr{E}_{l}\right\rangle+2 \pi j F+F X_{l}, l=1,2, \ldots, m, j \in \mathrm{Z}\right\},
$$

$\langle\cdot\rangle$ denotes the mean value on $\mathscr{B}$ 
The spectrum of $H_{c}^{\lambda}$ is purely essential and is given by

$$
\sigma\left(H_{c}^{\lambda}\right)=i F \mathfrak{I} \lambda+\mathrm{R} .
$$

Proof Taking into account that $X_{l}(k) \equiv\left\langle X_{l}\right\rangle$ is constant, the first statement is a well known result on the spectrum of the Wannier DB approximation. The second statement is obvious.

\section{Theorem 7.}

$$
\sigma_{\mathrm{ess}}\left(H_{F}^{\lambda}\right)=i F \mathfrak{I} \lambda+\mathrm{R} .
$$

Proof From the Weyl criterion (Theorem IV.5.35 in [17]) we have the stability of the essential spectrum if the perturbation $\tilde{X}^{\lambda}$ is relatively compact with respect to the unperturbed operator $H_{F}^{\mathrm{DB}, \lambda}$, that is if $\tilde{X}^{\lambda} R^{\lambda}(0, z)$ is a compact operator. Now, since the analytic continuation of an operator-valued function with compact values on the real axis is compact, it is sufficient to prove the compactness result when $\lambda$ is real, for instance $\lambda=0$. In particular, we prove the compactness of $X_{c, c}^{0} R_{c}^{0}(0, z)$ and the compactness of the other terms will follow in a similar way. Indeed, from the explicit expression of $R_{c}^{0}(0, z)$ (Sect. III.6 in [17]) for $0<\Im z$ (the case of $\mathfrak{I} z<0$ is similar) and from (II.16) for any $\eta \in \mathscr{H}_{c}$ we have that:

$$
\left(X_{c, c}^{0} R_{c}^{0}(0, z) \eta\right)(p)=\int_{\mathrm{R}} h(p, \tau) \eta(\tau) d \tau,
$$

where $h(p, \tau)$ is the measurable function in $L^{2}(\mathrm{R}, d p) \otimes L^{2}(\mathrm{R}, d \tau)$ given by:

$$
h(p, \tau)=\frac{i}{F} \sum_{L \neq 0} \chi_{[p,+\infty)}(\tau-L) \psi_{c}^{0}(p+L)\left[\psi_{c}^{0}(\tau)\right]^{-1} X_{c, c}^{L}(p),
$$

$\psi_{c}^{\lambda}(p)=\exp \left[i / F \int^{p}\left(\mathscr{E}_{m+1}(\tau)+F \lambda-z\right) d \tau\right]$ is a solution of $\left[H_{c}^{\lambda}-z\right] \psi=0$ and $\chi_{I}$ denotes the characteristic function on the set $I$. Now, from (II.18) we have:

$$
\int_{\mathrm{R}} d p \int_{\mathrm{R}} d \tau|h(p, \tau)|^{2} \leq C \int_{\mathrm{R}}\left(1+p^{2}\right)^{-2} d p<\infty,
$$

for some positive constant $C$, that is $X_{c, c}^{0} R_{c}^{0}(0, z)$ is of Hilbert-Schmidt type and thus compact.

Remark 8 As a first result we have that, from the analyticity of $H_{F}^{\lambda}$, from Theorem 7 and since the spectrum of $H_{F}$ is purely absolutely continuous for our class of potentials [5], the discrete spectrum of $H_{F}^{\lambda}$ :

$$
\sigma_{d}\left(H_{F}^{\lambda}\right)=\sigma\left(H_{F}^{\lambda}\right)-\sigma_{\text {ess }}\left(H_{F}^{\lambda}\right)
$$

is $\lambda$-independent and contained in the lower complex half-plane and it defines the resonances for $H_{F}$ in the stripe $F \mathfrak{I} \lambda<\mathfrak{I} z<0$.

Now, let $\Gamma$ be the positive clock-wise contour of a rectangle in the stripe $|\Im z| \leq F|\Im \lambda| / 2$ of length $2 \pi F$, height $F|\Im \lambda|$ and such that $\operatorname{dist}\left(\Gamma, \sigma\left(H_{d}\right)\right) \geq d=$ $\min \{\bar{F}|\Im \lambda| / 2, F \pi / m\}$; so it contains $m$ eigenvalues of $H_{d}^{\lambda}$, one for each Wannier ladder. Let $\varrho\left(H_{F}^{\lambda}(f)\right)$ be the resolvent set of $H_{F}^{\lambda}(f)$. We have the following result.

Theorem 9. Let $-\lambda_{0}<\mathfrak{I} \lambda<0$. There exists $F_{0}>0$ such that for any $F, 0<F \leq F_{0}$, $\Gamma \subset \varrho\left(H_{F}^{\lambda}(f)\right)$ for any $|f| \leq F$ 
Proof. We have to prove that the resolvent (III.2) is uniformly bounded for any $|f| \leq F$ and $z \in \Gamma$. In particular, the expression of $R_{d}^{\lambda}(f, z)$ for $z \in \Gamma$ is given by the partition method [16] and the resolvent formula:

$$
\begin{aligned}
R_{d}^{\lambda}(f, z) & =\left[H_{d}+f\left(X_{d, d}-X_{\mathrm{diag}}\right)-z-f X_{d, c}^{\lambda}\left[H_{c}^{\lambda}+f X_{c, c}^{\lambda}-z\right]^{-1} f X_{c, d}^{\lambda}\right]^{-1} \\
& =R_{d}(0, z)\left[1+Q_{d}(f, z)\right]^{-1}\left[1-f X_{d, c}^{\lambda} A^{\lambda}(f, z)\right]^{-1}
\end{aligned}
$$

where

$$
A^{\lambda}(f, z)=\left[1+Q_{c}^{\lambda}(f, z)\right]^{-1} R_{c}^{\lambda}(0, z) f X_{c, d}^{\lambda} R_{d}(0, z)\left[1+Q_{d}(f, z)\right]^{-1}
$$

is an operator from $\mathscr{H}_{d}$ to $\mathscr{H}_{c}, Q_{c}^{\lambda}(f, z)=R_{c}^{\lambda}(0, z) f X_{c, c}^{\lambda}$ is a bounded operator from $\mathscr{H}_{c}$ to $\mathscr{H}_{c}$ and $Q_{d}(f, z)=f\left(X_{d, d}-X_{\text {diag }}\right) R_{d}(0, z)$ is a bounded operator from $\mathscr{H}_{d}$ to $\mathscr{H}_{d}$. The other terms in (III.2) are given by:

$$
\begin{aligned}
R_{c}^{\lambda}(f, z) & =\left[1-A^{\lambda}(f, z) f X_{d, c}\right]^{-1}\left[1+Q_{c}^{\lambda}(f, z)\right]^{-1} R_{c}^{\lambda}(0, z), \\
S^{\lambda}(f, z) & =\mathscr{P} R^{\lambda}(f, z) \mathscr{P}^{\perp} \\
& =-R_{d}^{\lambda}(f, z) f X_{d, c}^{\lambda}\left[1+Q_{c}^{\lambda}(f, z)\right]^{-1} R_{c}^{\lambda}(0, z) \\
T^{\lambda}(f, z) & =\mathscr{P}^{\perp} R^{\lambda}(f, z) \mathscr{P} \\
& =-\left[1+Q_{c}^{\lambda}(f, z)\right]^{-1} R_{c}^{\lambda}(0, z) f X_{c, d}^{\lambda} R_{d}^{\lambda}(f, z) .
\end{aligned}
$$

We need of the following lemma.

Lemma 10. There exists $F_{0}>0$ such that for any $F, 0<F<F_{0}$, the following estimates uniformly hold for $z \in \Gamma$ and $|f| \leq F$ :

$$
\left\|R_{c}^{\lambda}(0, z) f X_{c, d}^{\lambda} R_{d}(0, z)\right\| \leq C
$$

and

$$
\left\|\left[1+Q_{c}^{\lambda}(f, z)\right]^{-1}\right\| \leq C, \quad\left\|\left[1+Q_{d}(f, z)\right]^{-1}\right\| \leq C
$$

for some positive constant $C$ Therefore, for any $0<F<F_{0}, A^{\lambda}(f, z)$ is a uniformly bounded operator from $\mathscr{H}_{d}$ to $\mathscr{H}_{c}$ for $z \in \Gamma$ and $|f| \leq F$ :

$$
\left\|A^{\lambda}(f, z)\right\| \leq C .
$$

Proof. Proof of (III.12): for $\xi=\left(\xi_{1}, \ldots, \xi_{m}\right) \in \mathscr{H}_{d}$ we have that (Sect. III.6 in [17]):

$$
\begin{aligned}
& {\left[R_{d}(0, z) \xi\right]_{l}(\tau)} \\
& \quad=-\frac{i}{F} \psi_{d, l}(\tau)\left\{\frac{1}{e^{\varrho_{l}}-1} \int_{\not \supset} \psi_{d, l}^{-1}(k) \xi_{l}(k) d k+\int_{0}^{\tau} \psi_{d, l}^{-1}(k) \xi_{l}(k) d k\right\},
\end{aligned}
$$

where $\varrho_{l}=i\left(z-E_{l, 0}\right) / F$ and

$$
\psi_{d, l}(\tau)=\exp \left[\frac{i}{F} \int_{0}^{\tau}\left(\mathscr{E}_{l}(k)+F X_{l}-z\right) d k\right]
$$


is a solution of $\left[H_{l}-z\right] \psi_{d, l}=0$. Hence, we obtain:

$$
\begin{aligned}
& {\left[R_{c}^{\lambda}(0, z) f X_{c, d}^{\lambda} R_{d}(0, z) \xi\right](p)} \\
& \quad=\sum_{l=1}^{m} \frac{f}{F^{2}} \psi_{c}^{\lambda}(p) \int_{p}^{+\infty}\left[\psi_{c}^{\lambda}(\tau)\right]^{-1} e^{\imath \tau \lambda} X_{c, d}^{l}(\tau) \psi_{d, l}(\tau) \\
& \quad \times\left[\frac{1}{e^{\varrho_{l}}-1} \int_{\not \beta} \psi_{d, l}^{-1}(k) \xi_{l}(k) d k+\int_{0}^{\tau} \psi_{d, l}^{-1}(k) \xi_{l}(k) d k\right] d \tau \\
& =\sum_{l=1}^{m}\left\{I_{l}+I I_{l}\right\} .
\end{aligned}
$$

\section{Denoting}

$$
\begin{aligned}
U_{l}(p) & =\int_{p}^{+\infty} e^{i \tau \lambda}\left[\psi_{c}^{\lambda}(\tau)\right]^{-1} X_{c, d}^{l}(\tau) \psi_{d, l}(\tau) d \tau \\
& =\int_{p}^{+\infty} X_{c, d}^{l}(\tau) \exp \left[i \tau X_{l}-\frac{i}{F} \int^{\tau}\left(\mathscr{E}_{m+1}(k)-\mathscr{E}_{l}(k)\right) d \kappa\right] d \tau
\end{aligned}
$$

we have that

$$
I_{l}=\frac{f}{F^{2}} \frac{1}{e^{\varrho_{l}}-1} \psi_{c}^{\lambda}(p) U_{l}(p) \int_{\not \supset} \psi_{d, l}^{-1}(k) \xi_{l}(k) d k
$$

and, integrating by parts,

$$
\begin{aligned}
I I_{l}= & \frac{f}{F^{2}} \psi_{c}^{\lambda}(p) U_{l}(p) \int_{0}^{p} \psi_{d, l}^{-1}(\tau) \xi_{l}(\tau) d \tau \\
& +\frac{f}{F^{2}} \psi_{c}^{\lambda}(p) \int_{p}^{+\infty} U_{l}(\tau) \psi_{d, l}^{-1}(\tau) \xi_{l}(\tau) d \tau
\end{aligned}
$$

Now, from (II.18) we have that for each $l$ the integral (III.17) is absolutely convergent and, by integrating by parts like in the stationary phase method, one obtains the following estimate:

$$
\left|U_{l}(p)\right| \leq F C\left(\chi_{(-\infty, 0]}(p)+\exp \left[-2 \lambda_{0}|p|\right]\right) .
$$

Hence, it follows that:

$$
\left\|I_{l}+I I_{l}\right\|_{\mathscr{H}_{c}} \leq C \frac{|f|}{F\left|e^{\varrho_{l}}-1\right|}\left\|\xi_{l}\right\|_{\mathscr{H}_{d}},
$$

and so the estimate (III.12) follows because $\left|\left(e^{\varrho_{l}}-1\right)^{-1}\right| \leq C$ for any $l$.

Proof of (III.13). The first estimate in (III.13) follows because:

$$
\left\|\left[Q_{c}^{\lambda}(f, z)\right]^{2}\right\| \leq\left\|Q_{c}^{\lambda}(f, z) f R_{c}^{\lambda}(0, z)\right\| \cdot\left\|X_{c, c}^{\lambda}\right\|=O(\sqrt{F})
$$


by the stationary phase method. Indeed, from (II.16) and by integrating by parts (see Sect. III.6 in [17] for the expression of $\left.R_{c}^{\lambda}(0, z)\right)$ we have that:

$$
\begin{aligned}
& {\left[f^{2} R_{c}^{\lambda}(0, z) X_{c, c}^{\lambda} R_{c}^{\lambda}(0, z) \eta\right](p)} \\
& =\frac{f^{2}}{F^{2}} \psi_{c}^{\lambda}(p) \sum_{L \in \mathrm{Z}, L \neq 0}\left\{-V_{l}(p) \int_{p}^{+\infty}\left[\psi_{c}^{\lambda}(\tau+L)\right]^{-1} \eta(\tau+L) d \tau\right. \\
& \left.\quad+\int_{p}^{+\infty} V_{L}(\tau)\left[\psi_{c}^{\lambda}(\tau+L)\right]^{-1} \eta(\tau+L) d \tau\right\}, \quad \eta \in \mathscr{H}_{c},
\end{aligned}
$$

where

$$
V_{L}(p)=e^{-i L z / F} \int_{p}^{+\infty} X_{c, c}^{L}(\tau) \exp \left[\frac{i}{F} \int_{\tau}^{\tau+L} \mathscr{E}_{m+1}(k) d k\right] d \tau
$$

Now, since $\mathscr{E}_{m+1}(-p)=\mathscr{E}_{m+1}(p)$ for real $p$, we have that, if $p \leq-L / 2$, then there exists $\tau_{0}=-L / 2 \in[p,+\infty)$ such that $\mathscr{E}_{m+1}\left(\tau_{0}+L\right)=\mathscr{E}_{m+1}\left(\tau_{0}\right)$ and so we can apply the stationary phase method. Here, the leading term of the asymptotic expansion of $V_{L}(p)$ given by the stationary phase method is bounded by $\sqrt{F} C(1+|L|)^{-1} \exp \left[-2|L| \lambda_{0}+L \Im z / F\right]$ for some positive constant $C$. Otherwise, for $p>-L / 2$ we have, by integrating by parts, the following estimate $\left|V_{L}(p)\right| \leq$ $F C|L| \exp \left[-2|L| \lambda_{0}+L \Im z / F\right] /(1+|p|)$. So, by both estimates, (III.22) follows.

In similar way one can prove that:

$$
\left\|Q_{d}^{2}\right\| \leq\left\|X_{d, d}-X_{\text {diag }}\right\| \cdot\left\|f R_{d}(0, z) Q_{d}(f, z)\right\|=O(F),
$$

and so the second estimate in (III.13) follows too. The proof of the lemma is so completed.

Now, we are ready to complete the proof of the theorem; indeed, from the above lemma and from the boundedness of $X_{d, c}^{\lambda}$ we have

$$
\left\|f X_{d, c}^{\lambda} A^{\lambda}(f, z)\right\| \leq C|f|<1 \quad \text { and } \quad\left\|A^{\lambda}(f, z) f X_{d, c}^{\lambda}\right\| \leq C|f|<1
$$

for any $|f| \leq F$, where $0<F<F_{0}$, for some $F_{0}>0$. Therefore, $R_{d}^{\lambda}(f, z)$ and $R_{c}^{l}(f, z)$ are uniformly bounded on $\Gamma$ for any $|f| \leq F$ and so, from (III.11), we have the uniform boundedness of the whole resolvent.

Now, let us state our main result:

Theorem 11. If the Hypothesis is satisfied, then there exists $F_{0}>0$ such that for any $F, 0<F<F_{0}$, the $m$ Wannier ladders of $H_{F}(0)$ become $m$ ladders of resonances $E_{l, \jmath}(f, F)$ of $H_{F}(f)$ for $f=F$. No other sharp resonance exists.

Proof. Let $\lambda \in \mathrm{C},-\lambda_{0}<\mathfrak{I} \lambda<0$, be fixed. Since $H_{F}^{\lambda}(f)$ is an analytic family of type $(A)$ for $|f| \leq F$, from the existence of $m$ isolated Wannier eigenvalues in $\Gamma$ for $f=0$ and from Theorem 9 the Kato-Rellich Theorem implies that

$$
\operatorname{dim} P_{F}^{\lambda}(f)=\operatorname{dim} P_{F}^{\lambda}(0)=m, \quad \text { for any }|f| \leq F,
$$


where $P_{F}^{\lambda}(f)$ is the eigenprojection on the domain enclosed by $\Gamma$ :

$$
P_{F}^{l}(f)=\frac{1}{2 \pi i} \oint_{\Gamma} R_{F}^{\lambda}(f, z) d z .
$$

Therefore, for $f=F$ we have the existence of $m$ eigenvalues for $H_{F}^{\lambda}$ contained in the domain enclosed by $\Gamma$ and from Theorem 7 and Remark 8 they represent $m$ resonances of $H_{F}=H_{F}(F)$.

Remark 12 The proof of existence of resonances we give is constructive and it directly comes from the Rayleigh-Schrödinger perturbation theory. In particular, the resonances $E_{l, j}(F)=E_{l, j}(F, F)$ are given by the degenerate perturbation equation:

$$
f(E)=\operatorname{det}\left(\oint_{\Gamma}\left\langle\psi_{d, l}, R_{d}(f, z) \psi_{d, l^{\prime}}\right\rangle z d z-E \oint_{\Gamma}\left\langle\psi_{d, l}, R_{d}(f, z) \psi_{d, l^{\prime}}\right\rangle d z\right)=0
$$

for $|f| \leq F$.

Remark 13. We recall the Nenciu method $[7,12,19,21]$ where, at the $n^{\text {th }}$ step, $H_{F}^{\lambda}=H_{F}^{\mathrm{DB}, \lambda}+F \tilde{X}^{\lambda}$ is redefined as

$$
H_{F}^{\lambda}=H_{F, n}^{\mathrm{DB}, \lambda}+F^{n+1} \tilde{X}_{n}^{\lambda} .
$$

Here, $H_{F, n}^{\mathrm{DB}, \lambda}$ has the same properties of $H_{F}^{\mathrm{DB}, \lambda}$, apart from a $F$ dependence of the redefined band functions, and $\tilde{X}_{n}^{\lambda}$ is still bounded. The above proof of existence works for (III.26) too and so each Wannier ladder obtained by $H_{F, n}^{\mathrm{DB}, \lambda}$ coincides with the ladder of resonances up to $O\left(F^{n+1}\right)$. This proves the existence of a real asymptotic expansion of the ladders coinciding with the Nenciu one (for an explicit computation of the second order term coinciding with the Nenciu one, see [14]). The asymptotic expansion of Nenciu does not directly come from the RayleighSchrödinger perturbation theory because of the dependence on $F$ in the perturbation coefficients. The width of the resonances is $O\left(F^{\infty}\right)$.

\section{Fermi Golden Rule and Estimate of the Width}

In the previous section we have proved the existence of ladders of resonances. In order to compute these resonances we consider the usual Rayleigh-Schrödinger formula. That is, let $E_{l, \jmath}$ be an isolated eigenvalue of $H_{F}^{\mathrm{DB}, \lambda}$ for $F \leq F_{0}$, we avoid crossing of resonances: $\left|E_{l, j}-E_{l^{\prime}, j^{\prime}}\right| \geq C F, \pi / m>C>0$. By mimicking the proof of Theorem 9 we can state that $\Gamma \subset \varrho\left(H_{F}^{\lambda}(f)\right)$ for any $|f| \leq F$, where $\Gamma$ is the circle surrounding $E_{l, 3}$ with radius $C F$.

Therefore, the Rayleigh-Schrödinger formula takes the form

$$
E_{l, j}(f, F)=\frac{\oint_{\Gamma}\left\langle\psi_{d, l}, R_{d}^{\lambda}(f, z) \psi_{d, l}\right\rangle_{\dddot{H}_{d}} z d z}{\oint_{\Gamma}\left\langle\psi_{d, l}, R_{d}^{\lambda}(f, z) \psi_{d, l}\right\rangle_{\mathscr{H}_{d}} d z}
$$


which gives, for $f=F$, the resonances $E_{l, 3}(F)=E_{l, 3}(F, F)$. Now, we have that the resonances coincide, up to $O\left(F^{2}\right)$, with the Wannier DB states and we obtain the second order perturbation approximation of $E_{l, j}(F)$ :

$$
E_{l, j}(F)=E_{l, j}^{2}(F)+O\left(F^{5 / 2}\right), \quad \text { as } \quad F \rightarrow 0 ;
$$

where the imaginary part of the second order term, if the Berry phase is absent (i.e. $\left.\left\langle X_{l}\right\rangle=0\right)$, is given by $[2,14]$ :

$$
\Im E_{l, \jmath}^{2}(F)=-\frac{F}{2}\left|\int_{\mathrm{R}} X_{d, c}^{l}(p) \exp \left[\frac{i}{F} \int_{0}^{p}\left(\mathscr{E}_{m+1}(\tau)-\mathscr{E}_{l}(\tau)\right) d \tau\right] d p\right|^{2} .
$$

Here, for $l=m$, the estimate of this integral is given by the saddle point method where we observe that the saddle points $z_{m}$ and $\bar{z}_{m}$ coincide with a polar singularity of $X_{d, c}^{m}(p)$ as well as with a branch point of square root type of the band functions. Indeed, in [14] we have proved that $z_{m}$ and $\bar{z}_{m}$ are simple poles of $X_{d, c}^{m}$ with residue $\pm 1 / 4$. Therefore, the stationary phase evaluation is determined by the minimal angle between two steepest descendent directions, i.e. $4 \pi / 3$, and the pole residue. Hence, we have that:

$$
\Im E_{m, j}^{2}(F)=-C F \exp \left[-\frac{i}{F} \oint_{\gamma_{m}} \mathscr{E}(p) d p\right] \cdot\left\{1+O\left(F^{2 / 3}\right)\right\},
$$

where $C=\pi^{2} / 18, \gamma_{m}$ is a clock-wise regular contour around a cut linking directly $z_{m}$ and $\bar{z}_{m}$ and $\mathscr{E}(p)$ is the energy function defined by $\mathscr{E}(m / 2+1)=\mathscr{E}_{m+1}(m / 2+1)$ on the sheet. This value of $C$ should be compared with the one of Buslaev and Dmitrieva [9, 14]: $C=1 / 2$, for the full behavior of $\Im E_{1, j}(F)$. If we are in the crossing restriction: $F$ such that $E_{l, \jmath}=E_{l^{\prime}, \jmath^{\prime}}$, we can study the crossing phenomena $[2,6,11]$.

Acknowledgements It is a pleasure to thank Professors François Bentosela, Vladimir Buslaev, Ludmila Dmitrieva and Alain Joye for many discussions on such problems This work is partially supported by Ministero dell'Università e della Ricerca Scientifica e Tecnologica

\section{References}

1 Agler, J, Froese, R : Existence of Stark ladder resonances Commun Math Phys 100, 161-171 (1985)

2 Avron, J: The lifetime of Wannier ladder state. Ann. Phys 143, 33-53 (1982)

3 Bastard, G., Brun, J.A, Ferreira, R: Electronic states in semiconductor heterostructes Solid States Physics (Academic Press) 44, 229-415 (1991)

4. Bentosela, F., Grecchi, V , Zironi, F : Approximate ladder of resonances in a semi-infinite crystal. J. Phys C: Solid State Phys 15, 7119-7131 (1982)

5 Bentosela, F, Carmona, R, Duclos, P, Simon, B., Souillard, B, Weder, R: Schrödinger operators with an electric field and random or deterministic potentials. Commun Math. Phys 88, 387-397 (1983)

6 Bentosela, F., Grecchi, V., Zironi, F.: Oscillations of Wannier resonances Phys Rev Lett 50, 84-86 (1983)

7. Bentosela, F.: Stark-Wannier resonant states Preprint CPT-90/P 2256 Luminy, 1990

8 Bentosela, F, Grecchi, V.: Stark-Wannier ladders Commun Math Phys. 142, 169-192 (1991) 
9 Buslaev, V.S., Dmitrieva, L A.: A Block electron in an external field. Leningrad Math J. 1, 287-320 (1990)

10 Combes, J.M., Hislop, P.D.: Stark ladder resonances for small electric fields. Commun. Math. Phys. 140, 291-320 (1991)

11 Ferrari, M., Grecchi, V., Zironi, F.: Stark-Wannier states and Bender-Wu singularities J Phys. C: Solid State Phys 18, 5825-5834 (1985)

12 Grecchi, V, Maioli, M., Sacchetti, A.: Horn of singularities for the Stark-Wannier ladders. J. Phys A: Math Gen. 24, 4275-4282 (1991)

13 Grecchi, V., Maioli, M, Sacchetti, A : Stark resonances in disordered systems. Commun. Math. Phys. 146, 231-240 (1992)

14. Grecchi, V, Maioli, M., Sacchetti, A : Wannier ladders and perturbation theory J Phys. A: Math Gen 26, L379-L384 (1993)

15 Herbst, I., Howland, J.: The Stark ladder and other one-dimensional external electric field problems. Commun Math Phys. 80, 23-42 (1981)

16. Howland, J.: The Livsic matrix and perturbation theory. J Math. Anal. Appl. 50, 415-437 (1975)

17. Kato, T.: Perturbation theory for linear operator. Berlin: Springer 1976

18. Marchenko, V.A., Ostrovsky, I.V : Approximation of periodic by finite-zone potentials. Selecta Math. Sov , 6, 101-136 (1987)

19 Nenciu, G.: Adiabatic theorem and spectral concentration. I. Arbitrary order spectral concentration for the Stark effect in atomic physics. Commun Math. Phys. 82, 121-135 (1981)

20 Reed, M., Simon, B.: Methods of modern Mathematical Physics. IV. Analysis of operators. New York: Academic Press 1978

21 Sacchetti, A : Asymptotic expansion of the Stark-Wannier state. Helv. Phys. Acta 65, 11-31 (1992)

22. Titchmars, E.C.: Eigenfunction expansions associated with second-order differential equations. II. Oxford: Clarendon Press 1958

23 Wannier, G H.: Wave functions and effective Hamiltonian for Bloch electrons in a electric field. Phys. Rev. 117, 432-439 (1960)

24. Zak, J.: Stark ladder in solid? Phys. Rev Lett 20, 1477-1481 (1968)

Communicated by B. Simon 